\title{
Effect of IBA (Indole-3-butyric acid) concentration on Success, Survival and Mortality of Pomegranate (Punica garanatum L.) Cutting
}

\author{
Basir Ahmad Ahmadi*, G. M. Waghmare and A. M. Bhosale \\ Department of Horticulture, VNMKV, parbhani, India \\ *Corresponding author
}

\section{A B S T R A C T}

Keywords

Indole-3-butyric acid, Pomegranate, FRBD, Cuttings

Article Info

Accepted:

12 December 2020

Available Online:

10 January 2021
The experiment" was carried out during the year 2017-18 at Department of Horticulture, Vasantrao Naik Marathwada Krishi Vidyapeeth, Parbhani on pomegranate (Punica granatum L.) cv. Bhagwa". The cuttings were taken from hardwood cuttings arising on trees of pomegranate cv. Bhagwa during the month of July. Experiment was laid out in Factorial Randomized Block Designed (FRBD) with 18 treatments and 3 factors which are replicated trice and no. of cuttings per treatment are 40 . Result revealed that, pomegranate cuttings of $1.5 \mathrm{~cm}$ diameter and $20 \mathrm{~cm}$ length when treated with IBA $3000 \mathrm{ppm}$ concentration solution for 15- 20 second gives significantly highest success percentage of pomegranate cuttings $\left(70.12 \%\right.$ ) was observed in treatment $\mathrm{T}_{1}$ i.e. (IBA $3000 \mathrm{ppm}$ ), highest survival percentage $(68.59 \%)$ was recorded in treatment $\mathrm{T}_{1}$ i.e. (IBA $3000 \mathrm{ppm}$ ) and lowest mortality percentage $(31.40 \%)$ of pomegranate cuttings was observed in treatment $\mathrm{T}_{1}$ i.e. (IBA $\left.3000 \mathrm{ppm}\right)$.

\section{Introduction}

Pomegranate (Punica granatum L.) belongs to family Punicaceae is native to Asia especially to Iran, Afghanistan and Himalayan region. It is a shrub that naturally tends to develop multiple trunks and has a bushy appearance. When domesticated, it is grown as a small tree that grows up to $5 \mathrm{~m}$, leaves have an oblanceolate shape with an apex and acuminate base. Mature leaves are green, entire, smooth and hairless; the flowers can appear solitary, pairs or cluster. In the most cases, the solitary flowers will appear as spurs along the branches while the clusters are terminal (Levin, 2006). Fruit is globular, possessing a smooth outer rind with juicy arils (Mars, 2000). The Bhagwa variety of pomegranate has attractive glossy red thick skin with high TSS this is very popular among the farmers and is cultivated in large area because of the good shelf life, effective skin and aril colour and tolerant to fruit cracking (Sheikh, 2002). The Bhagwa variety have good export potential major breakthrough in the cultivation of pomegranate such as resting period, use of plant hormone and pruning of plants to obtain better size fruit have contributed in increasing quality of pomegranate fruit to meet the preference of consumers in domestic and international market. 
Pomegranate could be propagated either sexually by seeds or vegetatively using stem cuttings and sometimes as layers or suckers or by grafting (Hartmann et al., 1997). However, in commercial propagation stem cuttings are widely used. (Antakya Hatay, 2009). Grafting and layering of pomegranate trees is rarely done, because many different types of grafts have not been successful enough for use in commercial production. Tissue culture is another method of production that calls for the growth of plant in a sterile environment using tissue seed, or cuttings. (Abdul Rehman and Al-Wasel, 1999).

Sprouting and rooting ability of cuttings is mainly depends upon the physiological maturity of the shoot and conditions where cuttings have been planted for sprouting and subsequent rooting (Purohit and Shekrappa, 1985) and various internal and external factors like seasons and concentration of endogenous and exogenous phytohormones (Arya et al., 1994). Plant growth regulators improve the rooting of cutting by stimulating the production of adventitious roots. Went (1934) first postulated that auxins initiate adventitious root formation in stem cuttings.

IBA is the most promising growth regulator inducing rooting quickly. Exogenous application of IBA accelerates the rate of rooting, increases final rooting percentage and number of roots. However, relatively high concentrations of IBA have been reported to be inhibitory to rooting (Leakey, 1990). It promotes root initiation, number of roots and shoots growth in number of ornamental and fruits plants. The rooting medium can have a major influence on the rooting capacity of cuttings (Hartmann et al., 2002).

\section{Materials and Methods}

The experiment" was carried out during the year 2017-18 at Department of Horticulture, Vasantrao Naik Marathwada Krishi Vidyapeeth, Parbhani on pomegranate (Punica granatum L.) cv. Bhagwa". Treatment Details:

\begin{tabular}{|c|c|c|c|c|c|}
\hline \multicolumn{2}{|c|}{ Factors: } & \multicolumn{2}{c|}{} \\
\hline \multicolumn{2}{|c|}{ Factor A: } \\
\multicolumn{2}{c|}{ Length of cutting } & \multicolumn{2}{c|}{$\begin{array}{c}\text { Factor B: } \\
\text { Diameter of cutting }\end{array}$} & \multicolumn{2}{c|}{$\begin{array}{c}\text { Factor C: } \\
\text { IBA concentration }\end{array}$} \\
\hline $\mathbf{L}_{\mathbf{1}}:$ & $10 \mathrm{~cm}$ & $\mathrm{D}_{1}:$ & $1 \mathrm{~cm}$ & $\mathrm{~T}_{1}:$ & $3000 \mathrm{ppm}$ \\
\hline $\mathbf{L}_{\mathbf{2}}:$ & $15 \mathrm{~cm}$ & $\mathrm{D}_{2}:$ & $1.5 \mathrm{~cm}$ & $\mathrm{~T}_{2}:$ & $4000 \mathrm{ppm}$ \\
\hline $\mathbf{L}_{\mathbf{3}}:$ & $20 \mathrm{~cm}$ & & & $\mathrm{~T}_{3}:$ & $5000 \mathrm{ppm}$ \\
\hline
\end{tabular}

\section{Observation recorded}

The experiment was replicated thrice with 40 cuttings in each treatment and a total of (2250) cuttings were tested. Five sprouted cuttings were selected randomly from each treatment of each replication. All given observations were recorded at intervals of 30 , 60 and 90 days after planting.

\section{Success percentage}

Percent success was counted 60 days after planting by counting the number of cuttings established and the results were expressed in percentage. It can be calculated by using following formula.

Success percentage $(\%)=\frac{\text { of live cuttings }}{\text { Total No. of cuttings }}$
----- x 100

Survival percentage

Survival percentage was counted 90 days after planting by counting the number of cuttings established and the results were expressed in percentage. 


\section{Mortality percentage}

Mortality percentage was counted 90 days after planting by counting the number of cuttings dead and the results were expressed in percentage.

\section{Results and Discussion}

The results obtained during the experiment were recorded and analysed statistically, presented under appropriate headings, subheadings and discussed with available literature.

\section{Success percentage}

The effect diameter of cutting (D), length of cutting (L), IBA concentrations (T) and their interactions on success percentage of pomegranate cuttings presented in Table 1.

\section{Effect of cutting diameter (D)}

Significantly highest success percentage $(70.67 \%)$ was recorded in treatment $\mathrm{D}_{2}$ i.e. (1.5 cm diameter) and lowest (65.13\%) was observed in $\mathrm{D}_{1}$ i.e. (1 $\mathrm{cm}$ diameter).

\section{Effect of cutting length $(\mathrm{L})$}

Significantly, highest success percentage (70.85\%) was recorded with $\mathrm{L}_{3}$ i.e. $(20 \mathrm{~cm}$ length) and treatment $\mathrm{L}_{2}$ i.e. (15 cm length) recorded lowest $(68.29 \%)$ success percentage. might be due to the fact that, large sized cuttings might have got sufficient food material and hormones leading to more survival (Kaur and Kaur 2018).

\section{IBA concentration ( $T$ )}

With respect to IBA concentrations, significantly highest success percentage of pomegranate cuttings $(70.12 \%)$ was observed in treatment $\mathrm{T}_{1}$ i.e. (IBA $3000 \mathrm{ppm}$ ) and significantly lowest success percentage (67.60 $\%$ ) was recorded with treatment $\mathrm{T}_{2}$ i.e. (IBA $4000 \mathrm{ppm}$ ). This might be due to IBA which regulate different aspects of growth and development of plant in terms of cell division, cell elongation and differentiation which leads to formation of great quantity of roots and shoots making great plant and higher plant survival (Davis 1996).

\section{Interaction effect of $(L \times D)$}

The interaction of length and diameter of cutting shows significant variation on success percentage. Significantly, highest success percentage $(75.98 \%)$ was recorded in interaction of $\mathrm{L}_{3} \mathrm{D}_{2}$ i.e. (length $20 \mathrm{~cm}$ and diameter $1.5 \mathrm{~cm})$ and lowest success percentage $(61.48 \%)$ was observed in interaction of $\mathrm{L}_{1} \mathrm{D}_{1}$ i.e. (length $10 \mathrm{~cm}$ and 1 $\mathrm{cm}$ diameter).

\section{Interaction effect of $(T \times D)$}

Significantly, highest success percentage $(72.87 \%)$ was recorded in interaction of $\mathrm{T}_{1} \mathrm{D}_{2}$ i.e. (IBA $3000 \mathrm{ppm}$ and $1.5 \mathrm{~cm}$ diameter) which was at par with interaction of $\mathrm{T}_{3} \mathrm{D}_{2}$ $(71.32 \%)$ and lowest success percentage $(60.65 \%)$ was observed in interaction of $\mathrm{T}_{3} \mathrm{D}_{1}$ i.e. (IBA $5000 \mathrm{ppm}$ and $1 \mathrm{~cm}$ diameter).

\section{Interaction effect of $(T \times L)$}

The interaction of $\mathrm{T}_{1} \mathrm{~L}_{3}$ i.e. (IBA $3000 \mathrm{ppm}$ and $20 \mathrm{~cm}$ length) recorded significantly highest success percentage $(75.43 \%)$, which was followed by interactions of $\mathrm{T}_{3} \mathrm{~L}_{3}(72.51$ $\%)$ and $\mathrm{T}_{1} \mathrm{~L}_{2}(69.10 \%)$, while the lowest success percentage $(62.10 \%)$ was observed in interaction of $\mathrm{T}_{2} \mathrm{~L}_{1}$ i.e. (IBA $4000 \mathrm{ppm}$ and 10 $\mathrm{cm}$ length). However, the interaction effect of IBA concentrations, diameter and length of cuttings $(T \times D \times L)$ do not show significant variation on success percentage of pomegranate cuttings. 
Table.1 Effect of cutting size and IBA concentration on success and survival percentage of pomegranate cuttings

\begin{tabular}{|c|c|c|}
\hline Treatments & $\begin{array}{c}\text { Success percentage } \\
\text { 90DAP }\end{array}$ & $\begin{array}{c}\text { Survival percentage } \\
\text { 90DAP }\end{array}$ \\
\hline \multicolumn{3}{|c|}{ Diameter of cutting (D) } \\
\hline $\mathrm{D}_{1}$ & $65.13 \quad(40.96)$ & $63.60 \quad(39.78)$ \\
\hline $\mathrm{D}_{2}$ & $70.67 \quad(45.27)$ & $69.14 \quad(44.04)$ \\
\hline S.E N \pm & 1.03 & 1.03 \\
\hline C.D. at $5 \%$ & 3.09 & 3.09 \\
\hline \multicolumn{3}{|c|}{ Length of cutting (L) } \\
\hline $\mathrm{L}_{1}$ & $64.57 \quad(40.57)$ & $63.03 \quad(39.38)$ \\
\hline $\mathrm{L}_{2}$ & $68.29 \quad(43.17)$ & $66.76 \quad(42.00)$ \\
\hline $\mathrm{L}_{3}$ & $70.85 \quad(45.62)$ & $69.31 \quad(44.36)$ \\
\hline S.E N \pm & 1.15 & 1.15 \\
\hline C.D. at $5 \%$ & 3.45 & 3.45 \\
\hline \multicolumn{3}{|c|}{ IBA concentration $(\mathrm{T})$} \\
\hline $\mathrm{T}_{1}$ & $70.12 \quad(44.94)$ & $68.59 \quad(43.68)$ \\
\hline $\mathrm{T}_{2}$ & $67.60 \quad(42.82)$ & $66.06 \quad(41.59)$ \\
\hline $\mathrm{T}_{3}$ & $68.98 \quad(43.89)$ & $67.45 \quad(42.66)$ \\
\hline S.E N \pm & 1.15 & 1.15 \\
\hline C.D. at $5 \%$ & 3.45 & 3.45 \\
\hline \multicolumn{3}{|c|}{ Interaction $(\mathrm{L} \times \mathrm{D})$} \\
\hline $\mathrm{L}_{1} \mathrm{D}_{1}$ & $61.48 \quad(38.23)$ & $59.95 \quad(37.10)$ \\
\hline $\mathrm{L}_{2} \mathrm{D}_{1}$ & $68.21 \quad(43.11)$ & $66.67 \quad(41.93)$ \\
\hline $\mathrm{L}_{3} \mathrm{D}_{1}$ & $65.71 \quad(41.54)$ & $64.17 \quad(40.31)$ \\
\hline $\mathrm{L}_{1} \mathrm{D}_{2}$ & $67.65 \quad(42.90)$ & $66.12 \quad(41.66)$ \\
\hline $\mathrm{L}_{2} \mathrm{D}_{2}$ & $68.37 \quad(43.23)$ & $66.84 \quad(42.06)$ \\
\hline $\mathrm{L}_{3} \mathrm{D}_{2}$ & $75.98 \quad(49.70)$ & $74.45 \quad(48.40)$ \\
\hline S.E N \pm & 1.18 & 1.18 \\
\hline C.D. at $5 \%$ & 3.54 & 3.54 \\
\hline \multicolumn{3}{|c|}{ Interaction $(\mathrm{T} \times \mathrm{D})$} \\
\hline $\mathrm{T}_{1} \mathrm{D}_{1}$ & $67.37 \quad(42.62)$ & $64.84 \quad(41.44)$ \\
\hline $\mathrm{T}_{1} \mathrm{D}_{2}$ & $72.87 \quad(47.27)$ & $71.34 \quad(45.93)$ \\
\hline $\mathrm{T}_{2} \mathrm{D}_{1}$ & $67.37 \quad(42.74)$ & $65.84 \quad(41.48)$ \\
\hline $\mathrm{T}_{2} \mathrm{D}_{2}$ & $67.82 \quad(42.89)$ & $66.28 \quad(41.70)$ \\
\hline $\mathrm{T}_{3} \mathrm{D}_{1}$ & $60.65 \quad(37.53)$ & $59.12 \quad(36.42)$ \\
\hline $\mathrm{T}_{3} \mathrm{D}_{2}$ & $71.32 \quad(45.67)$ & $69.78 \quad(44.49)$ \\
\hline S.E N \pm & 1.18 & 1.18 \\
\hline C.D. at $5 \%$ & 3.55 & 3.55 \\
\hline
\end{tabular}

* figures in parenthesis indicate the arc sine values 


\begin{tabular}{|c|c|c|}
\hline Interaction $(\mathrm{L} \times \mathrm{T})$ & 90DAP & 90DAP \\
\hline $\mathrm{T}_{1} \mathrm{~L}_{1}$ & $65.85 \quad(41.63)$ & $64.31 \quad(40.45)$ \\
\hline$T_{1} L_{2}$ & $69.10 \quad(43.87)$ & $67.56 \quad(42.69)$ \\
\hline $\mathrm{T}_{1} \mathrm{~L}_{3}$ & $75.43 \quad(49.34)$ & $73.90 \quad(47.92)$ \\
\hline $\mathrm{T}_{2} \mathrm{~L}_{1}$ & $62.10 \quad(38.80)$ & $60.56 \quad(37.58)$ \\
\hline $\mathrm{T}_{2} \mathrm{~L}_{2}$ & $68.18 \quad(43.06)$ & $66.65 \quad(41.87)$ \\
\hline $\mathrm{T}_{2} \mathrm{~L}_{3}$ & $64.60 \quad(40.94)$ & $63.06 \quad(39.83)$ \\
\hline $\mathrm{T}_{3} \mathrm{~L}_{1}$ & $65.76 \quad(41.27)$ & $64.23 \quad(40.11)$ \\
\hline $\mathrm{T}_{3} \mathrm{~L}_{2}$ & $67.60 \quad(42.58)$ & $66.06 \quad(41.43)$ \\
\hline $\mathrm{T}_{3} \mathrm{~L}_{3}$ & $72.51 \quad(46.58)$ & $70.98 \quad(45.32)$ \\
\hline S.E N \pm & 1.04 & 1.04 \\
\hline C.D. at $5 \%$ & 3.12 & 3.12 \\
\hline \multicolumn{3}{|l|}{ Interaction ( $\mathrm{T} \times \mathrm{D} \times \mathrm{L})$} \\
\hline $\mathrm{T}_{1} \mathrm{D}_{1} \mathrm{~L}_{1}$ & $61.02 \quad(37.95)$ & $59.73 \quad(36.93)$ \\
\hline $\mathrm{T}_{1} \mathrm{D}_{1} \mathrm{~L}_{2}$ & $70.43 \quad(44.98)$ & $68.90 \quad(43.77)$ \\
\hline $\mathrm{T}_{1} \mathrm{D}_{1} \mathrm{~L}_{3}$ & $71.69 \quad(46.02)$ & $69.00 \quad(43.52)$ \\
\hline $\mathrm{T}_{1} \mathrm{D}_{2} \mathrm{~L}_{1}$ & $72.12 \quad(46.52)$ & $68.52 \quad(43.95)$ \\
\hline $\mathrm{T}_{1} \mathrm{D}_{2} \mathrm{~L}_{2}$ & $67.76 \quad(42.76)$ & $66.23 \quad(41.60)$ \\
\hline $\mathrm{T}_{1} \mathrm{D}_{2} \mathrm{~L}_{3}$ & $80.43 \quad(53.73)$ & $78.90 \quad(52.23)$ \\
\hline $\mathrm{T}_{2} \mathrm{D}_{1} \mathrm{~L}_{1}$ & $62.26 \quad(38.34)$ & $58.12 \quad(37.07)$ \\
\hline $\mathrm{T}_{2} \mathrm{D}_{1} \mathrm{~L}_{2}$ & $67.58 \quad(42.18)$ & $65.56 \quad(41.01)$ \\
\hline $\mathrm{T}_{2} \mathrm{D}_{1} \mathrm{~L}_{3}$ & $73.76 \quad(47.70)$ & $72.23 \quad(46.76)$ \\
\hline $\mathrm{T}_{2} \mathrm{D}_{2} \mathrm{~L}_{1}$ & $63.93 \quad(39.26)$ & $61.40 \quad(38.09)$ \\
\hline $\mathrm{T}_{2} \mathrm{D}_{2} \mathrm{~L}_{2}$ & $69.26 \quad(43.95)$ & $67.73 \quad(42.73)$ \\
\hline $\mathrm{T}_{2} \mathrm{D}_{2} \mathrm{~L}_{3}$ & $71.26 \quad(45.47)$ & $69.73 \quad(44.27)$ \\
\hline $\mathrm{T}_{3} \mathrm{D}_{1} \mathrm{~L}_{1}$ & $61.93 \quad(38.41)$ & $60.40 \quad(37.30)$ \\
\hline $\mathrm{T}_{3} \mathrm{D}_{1} \mathrm{~L}_{2}$ & $67.10 \quad(42.18)$ & $65.56 \quad(41.01)$ \\
\hline $\mathrm{T}_{3} \mathrm{D}_{1} \mathrm{~L}_{3}$ & $52.93 \quad(31.99)$ & $51.40 \quad(30.96)$ \\
\hline $\mathrm{T}_{3} \mathrm{D}_{2} \mathrm{~L}_{1}$ & $69.60 \quad(44.13)$ & $68.06 \quad(42.92)$ \\
\hline $\mathrm{T}_{3} \mathrm{D}_{2} \mathrm{~L}_{2}$ & $68.10 \quad(42.99)$ & $66.70 \quad(41.86)$ \\
\hline $\mathrm{T}_{3} \mathrm{D}_{2} \mathrm{~L}_{3}$ & $76.26 \quad(49.89)$ & $74.73 \quad(48.71)$ \\
\hline S.E N \pm & 4.30 & 4.30 \\
\hline $\mathrm{CD}$ at $5 \%$ & NS & NS \\
\hline \multicolumn{3}{|l|}{ Treatment details } \\
\hline \multirow[t]{2}{*}{$\mathrm{D}_{1}$-Diameter $1 \mathrm{~cm}$} & $\mathrm{~L}_{1}$ - Length $10 \mathrm{~cm}$ & $\mathrm{~T}_{1}-3000 \mathrm{ppm} \mathrm{IBA}$ \\
\hline & $\mathrm{L}_{2}$ - Length $15 \mathrm{~cm}$ & $\mathrm{~T}_{2^{-}}-4000 \mathrm{ppm} \mathrm{IBA}$ \\
\hline $\mathrm{D}_{2}$-Dimeter $1.5 \mathrm{~cm}$ & $\mathrm{~L}_{3}$ - Length $20 \mathrm{~cm}$ & $\mathrm{~T}_{3^{-}}-5000 \mathrm{ppm} \mathrm{IBA}$ \\
\hline
\end{tabular}

* figures in parenthesis indicate the arc sine values 
Table.2 Effect of cutting size and IBA concentration on mortality percentage of pomegranate cuttings

\begin{tabular}{|c|c|}
\hline \multirow[t]{2}{*}{ Treatments } & \multirow{2}{*}{$\begin{array}{c}\text { Mortality percentage } \\
\text { 90DAP }\end{array}$} \\
\hline & \\
\hline \multicolumn{2}{|c|}{ Diameter of cutting (D) } \\
\hline $\mathrm{D}_{1}$ & $36.39 \quad(21.45)$ \\
\hline $\mathrm{D}_{2}$ & $30.85 \quad(18.03)$ \\
\hline S.E N \pm & 1.03 \\
\hline C.D. at $5 \%$ & 3.09 \\
\hline \multicolumn{2}{|c|}{ Length of cutting (L) } \\
\hline $\mathrm{L}_{1}$ & $36.96 \quad(21.80)$ \\
\hline $\mathrm{L}_{2}$ & $33.23 \quad(19.44)$ \\
\hline $\mathrm{L}_{3}$ & $30.68 \quad(17.97)$ \\
\hline S.E N \pm & 1.15 \\
\hline C.D. at $5 \%$ & 3.45 \\
\hline \multicolumn{2}{|c|}{ IBA concentration $(\mathrm{T})$} \\
\hline $\mathrm{T}_{1}$ & $31.40 \quad(18.38)$ \\
\hline $\mathrm{T}_{2}$ & $33.93 \quad(19.92)$ \\
\hline $\mathrm{T}_{3}$ & $32.28 \quad(18.56)$ \\
\hline S.E N \pm & 1.15 \\
\hline C.D. at $5 \%$ & 3.45 \\
\hline \multicolumn{2}{|c|}{ Interaction (L x D) } \\
\hline $\mathrm{L}_{1} \mathrm{D}_{1}$ & $40.04 \quad(23.73)$ \\
\hline $\mathrm{L}_{2} \mathrm{D}_{1}$ & $33.32 \quad(19.49)$ \\
\hline $\mathrm{L}_{3} \mathrm{D}_{1}$ & $35.82 \quad(21.12)$ \\
\hline $\mathrm{L}_{1} \mathrm{D}_{2}$ & $33.87 \quad(19.86)$ \\
\hline $\mathrm{L}_{2} \mathrm{D}_{2}$ & $33.15 \quad(19.39)$ \\
\hline $\mathrm{L}_{3} \mathrm{D}_{2}$ & $25.54 \quad(14.82)$ \\
\hline S.E N \pm & 1.18 \\
\hline C.D. at $5 \%$ & 3.54 \\
\hline \multicolumn{2}{|c|}{ Interaction (T x D) } \\
\hline $\mathrm{T}_{1} \mathrm{D}_{1}$ & $34.15 \quad(20.04)$ \\
\hline $\mathrm{T}_{1} \mathrm{D}_{2}$ & $28.65 \quad(16.71)$ \\
\hline $\mathrm{T}_{2} \mathrm{D}_{1}$ & $34.35 \quad(20.25)$ \\
\hline $\mathrm{T}_{2} \mathrm{D}_{2}$ & $33.71 \quad(19.75)$ \\
\hline $\mathrm{T}_{3} \mathrm{D}_{1}$ & $40.87 \quad(24.21)$ \\
\hline $\mathrm{T}_{3} \mathrm{D}_{2}$ & $29.21 \quad(17.12)$ \\
\hline S.E N \pm & 1.18 \\
\hline C.D. at $5 \%$ & 3.55 \\
\hline
\end{tabular}

* figures in parenthesis indicate the arc sine values 


\begin{tabular}{|c|c|c|}
\hline \multirow{2}{*}{\multicolumn{2}{|c|}{$\begin{array}{c}\text { Interaction }(\mathrm{L} \times \mathrm{T}) \\
\mathrm{T}_{1} \mathrm{~L}_{1}\end{array}$}} & 90DAP \\
\hline & & $35.69 \quad(21.01)$ \\
\hline \multicolumn{2}{|c|}{$\mathrm{T}_{1} \mathrm{~L}_{2}$} & $32.44 \quad(18.96)$ \\
\hline \multicolumn{2}{|c|}{$\mathrm{T}_{1} \mathrm{~L}_{3}$} & $26.10 \quad(15.16)$ \\
\hline \multicolumn{2}{|c|}{$\mathrm{T}_{2} \mathrm{~L}_{1}$} & $39.44 \quad(23.38)$ \\
\hline \multicolumn{2}{|c|}{$\mathrm{T}_{2} \mathrm{~L}_{2}$} & $33.35 \quad(19.49)$ \\
\hline \multicolumn{2}{|c|}{$\mathrm{T}_{2} \mathrm{~L}_{3}$} & $29.02 \quad(16.88)$ \\
\hline \multicolumn{2}{|c|}{$\mathrm{T}_{3} \mathrm{~L}_{1}$} & $35.76 \quad(21.00)$ \\
\hline \multicolumn{2}{|c|}{$T_{3} L_{2}$} & $33.93 \quad(19.85)$ \\
\hline \multicolumn{2}{|c|}{$\mathrm{T}_{3} \mathrm{~L}_{3}$} & $28.25 \quad(17.52)$ \\
\hline \multicolumn{2}{|c|}{ S.E N \pm} & 1.04 \\
\hline C.D. at $5 \%$ & & 3.12 \\
\hline \multicolumn{3}{|c|}{ Interaction $(\mathrm{T} \times \mathrm{D} \times \mathrm{L})$} \\
\hline \multicolumn{2}{|c|}{$\mathrm{T}_{1} \mathrm{D}_{1} \mathrm{~L}_{1}$} & $40.27 \quad(23.84)$ \\
\hline \multicolumn{2}{|c|}{$\mathrm{T}_{1} \mathrm{D}_{1} \mathrm{~L}_{2}$} & $31.10 \quad(18.16)$ \\
\hline \multicolumn{2}{|c|}{$\mathrm{T}_{1} \mathrm{D}_{1} \mathrm{~L}_{3}$} & $31.00 \quad(18.13)$ \\
\hline \multicolumn{2}{|c|}{$\mathrm{T}_{1} \mathrm{D}_{2} \mathrm{~L}_{1}$} & $31.48 \quad(18.18)$ \\
\hline \multicolumn{2}{|c|}{$\mathrm{T}_{1} \mathrm{D}_{2} \mathrm{~L}_{2}$} & $33.77 \quad(19.77)$ \\
\hline \multicolumn{2}{|c|}{$\mathrm{T}_{1} \mathrm{D}_{2} \mathrm{~L}_{3}$} & $21.10 \quad(12.19)$ \\
\hline \multicolumn{2}{|c|}{$\mathrm{T}_{2} \mathrm{D}_{1} \mathrm{~L}_{1}$} & $41.88 \quad(23.96)$ \\
\hline \multicolumn{2}{|c|}{$\mathrm{T}_{2} \mathrm{D}_{1} \mathrm{~L}_{2}$} & $34.44 \quad(20.15)$ \\
\hline \multicolumn{2}{|c|}{$\mathrm{T}_{2} \mathrm{D}_{1} \mathrm{~L}_{3}$} & $27.77 \quad(16.13)$ \\
\hline \multicolumn{2}{|c|}{$\mathrm{T}_{2} \mathrm{D}_{2} \mathrm{~L}_{1}$} & $38.60 \quad(22.79)$ \\
\hline \multicolumn{2}{|c|}{$\mathrm{T}_{2} \mathrm{D}_{2} \mathrm{~L}_{2}$} & $32.27 \quad(18.84)$ \\
\hline \multicolumn{2}{|c|}{$\mathrm{T}_{2} \mathrm{D}_{2} \mathrm{~L}_{3}$} & $30.27 \quad(17.62)$ \\
\hline \multicolumn{2}{|c|}{$\mathrm{T}_{3} \mathrm{D}_{1} \mathrm{~L}_{1}$} & $39.60 \quad(23.38)$ \\
\hline \multicolumn{2}{|c|}{$\mathrm{T}_{3} \mathrm{D}_{1} \mathrm{~L}_{2}$} & $34.44 \quad(20.15)$ \\
\hline $\mathrm{T}_{3} \mathrm{D}_{1} \mathrm{~L}$ & & $48.60 \quad(29.10)$ \\
\hline $\mathrm{T}_{3} \mathrm{D}_{2} \mathrm{~L}$ & & $31.94 \quad(18.62)$ \\
\hline $\mathrm{T}_{3} \mathrm{D}_{2} \mathrm{~L}$ & & $33.30 \quad(19.56)$ \\
\hline $\mathrm{T}_{3} \mathrm{D}_{2} \mathrm{~L}$ & & $24.27 \quad(13.26)$ \\
\hline S.E N & & 4.30 \\
\hline $\mathrm{CD}$ at 5 & & NS \\
\hline Treatment detai & & \\
\hline $\mathrm{D}_{1}$-Diameter 1 & $\mathrm{~L}_{1}$ - Length $10 \mathrm{~cm}$ & $\mathrm{~T}_{1}-3000 \mathrm{ppm} \mathrm{IBA}$ \\
\hline $\mathrm{cm}$ & $\mathrm{L}_{2}$ - Length $15 \mathrm{~cm}$ & $\mathrm{~T}_{2}-4000 \mathrm{ppm} \mathrm{IBA}$ \\
\hline $\begin{array}{c}\mathrm{D}_{2} \text {-Dimeter } 1.5 \\
\mathrm{~cm}\end{array}$ & $\mathrm{~L}_{3}$ - Length $20 \mathrm{~cm}$ & $\mathrm{~T}_{3^{-}}-5000 \mathrm{ppm} \mathrm{IBA}$ \\
\hline
\end{tabular}

* figures in parenthesis indicate the arc sine values 


\section{Survival percentage}

The results on survival percentage of pomegranate cutting presented in Table 1 . As influenced by diameter of cutting (D), length of cutting (L), IBA concentrations (T) and their interactions.

\section{Effect of cutting diameter (D)}

The results revealed that, cutting diameter $\mathrm{D}_{2}$ i.e. $(1.5 \mathrm{~cm}$ diameter $)$ recorded highest survival percentage $(69.14 \%)$ whereas lowest survival percentage $(63.60 \%)$ was observed in $\mathrm{D}_{1}$ i.e. $(1 \mathrm{~cm}$ diameter $)$.

\section{Effect of cutting length $(\mathrm{L})$}

Among the length of cuttings, the highest success percentage $(69.31 \%)$ was recorded with $\mathrm{L}_{3}$ i.e. (20 cm length) and lowest success percentage $(63.03 \%)$ was found in $\mathrm{L}_{1}$ i.e. $(10$ $\mathrm{cm}$ length). This might be due to better development of root system with good quality root and shoot parameters enabling the rooted cuttings to make better growth and there by accounted the highest survivability. Sharma et al., (2009).

\section{IBA concentration $(T)$}

The results indicate that IBA concentration have positive effect on survival percentage of pomegranate cutting. Significantly, highest survival percentage $(68.59 \%)$ was recorded in treatment $\mathrm{T}_{1}$ i.e. (IBA $3000 \mathrm{ppm}$ ) and lowest survival percentage $(66.06 \%)$ was observed in $\mathrm{T}_{2}$ i.e. (IBA $4000 \mathrm{ppm}$ ). Davis (1996). This might be due to the fact that, IBA hormones have shown to regulate different aspects of growth and development of plant in terms of cell division, elongation and differentiation which leads to formation of great quantity of roots and shoots making great plant and higher plant survival.

\section{Interaction effect of $(L \times D)$}

The interaction of length and diameter of cutting shows significant effect on survival percentage. Significantly, highest survival percentage $(74.45 \%)$ of cutting was observed in interaction of $\mathrm{L}_{3} \mathrm{D}_{2}$ i.e. (length $20 \mathrm{~cm}$ and diameter $1.5 \mathrm{~cm}$ ) and lowest percentage $(59.95 \%)$ was observed in interaction of $\mathrm{L}_{1} \mathrm{D}_{1}$ i.e. (length $10 \mathrm{~cm}$ and diameter $1 \mathrm{~cm}$ ).

\section{Interaction effect of $(T \times D)$}

Significantly highest survival percentage (71.34 \%) of pomegranate cuttings was observed in interaction of $\mathrm{T}_{1} \mathrm{D}_{2}$ i.e. (IBA 3000 $\mathrm{ppm}$ and $1.5 \mathrm{~cm}$ diameter) which was followed by interactions of $\mathrm{T}_{3} \mathrm{D}_{2}(69.78 \%)$. whereas lowest survival percentage $(59.12 \%)$ was observed in interaction of $\mathrm{T}_{3} \mathrm{D}_{1}$ i.e. (IBA $5000 \mathrm{ppm}$ and $1 \mathrm{~cm}$ diameter).

\section{Interaction effect of $(T \times L)$}

The interaction effect of length of cutting and IBA concentration on survival percentage shows significant variation. The interaction $\mathrm{T}_{1} \mathrm{~L}_{3}$ i.e. (IBA $3000 \mathrm{ppm}$ and $20 \mathrm{~cm}$ length) recorded significantly highest survival percentage $(73.90 \%)$ which was followed by the interaction of $\mathrm{T}_{3} \mathrm{~L}_{3}(70.98 \%)$ while lowest survival percentage $(60.56 \%)$ was observed with interaction of $\mathrm{T}_{2} \mathrm{~L}_{1}$ i.e. (IBA $4000 \mathrm{ppm}$ and $10 \mathrm{~cm}$ length). However, the interaction of IBA concentrations, length and diameter of cuttings $(\mathrm{T} \times \mathrm{D} \times \mathrm{L})$ do not show significant variation on survival percentage of pomegranate cuttings.

\section{Mortality percentage}

The present study shows significant variation on mortality percentage in response to diameter of cutting (D), length of cutting (L), IBA concentrations ( $T$ ) and their interactions are presented in Table 2. 


\section{Effect of cutting diameter (D)}

The result obtained from the study revealed that, lowest mortality percentage $(30.85 \%)$ was recorded in $\mathrm{D}_{2}$ i.e. $(1.5 \mathrm{~cm}$ diameter) and highest mortality percentage $(36.39 \%)$ was observed in $\mathrm{D}_{1}$ i.e. $(1 \mathrm{~cm}$ diameter).

\section{Effect of cutting length ( $L)$}

The length of cutting had significant effect on mortality percentage. The lowest mortality percentage $(30.68 \%)$ was observed in treatment $\mathrm{L}_{3}$ i.e. $(20 \mathrm{~cm}$ length) and highest mortality percentage $(36.96 \%)$ was observed in $\mathrm{L}_{1}$ i.e. (10 $\mathrm{cm}$ length). this might be due to large sized cuttings, which are less liable to desiccation in comparison to small sized cuttings consequently, the former resulted in lowest mortality, Rana and Sood (2012).

\section{IBA concentration ( $T$ )}

Significantly, lowest mortality percentage $(31.40 \%)$ of pomegranate cuttings was observed in treatment $\mathrm{T}_{1}$ i.e. (IBA $3000 \mathrm{ppm}$ ) and highest $(33.93 \%)$ was recorded with treatment $\mathrm{T}_{2}$ i.e. (IBA $4000 \mathrm{ppm}$ ).

\section{Interaction effect of $(\mathbf{L} \times \mathbf{D})$}

The interaction of length and diameter of cutting on mortality percentage was found to be significant. Significantly lowest mortality percentage $(25.54 \%)$ was recorded with interaction of $\mathrm{L}_{3} \mathrm{D}_{2}$ i.e. (length $20 \mathrm{~cm}$ and 1.5 $\mathrm{cm}$ diameter) and highest mortality percentage $(40.04 \%)$ was observed in interaction of $\mathrm{L}_{1} \mathrm{D}_{1}$ i.e. (length $10 \mathrm{~cm}$ and 1 $\mathrm{cm}$ diameter).

\section{Interaction effect of $(T \times D)$}

Significantly, lowest mortality percentage $(28.65 \%)$ of pomegranate cuttings was recorded in interaction of $\mathrm{T}_{1} \mathrm{D}_{2}$ i.e. (IBA 3000 ppm and $1.5 \mathrm{~cm}$ diameter) which was followed by interactions of $\mathrm{T}_{3} \mathrm{D}_{2}(29.21 \%)$ whereas highest mortality percentage $(40.87$ $\%$ ) was observed by interaction of $\mathrm{T}_{3} \mathrm{D}_{1}$ i.e. (IBA $5000 \mathrm{ppm}$ and $1 \mathrm{~cm}$ diameter).

\section{Interaction effect of $(T \times L)$}

The interaction of $\mathrm{T}_{1} \mathrm{~L}_{3}$ i.e. (IBA $3000 \mathrm{ppm}$ and $20 \mathrm{~cm}$ length) was recorded significantly lowest mortality percentage $(26.10 \%)$ which was statistically at par with interaction of $\mathrm{T}_{3} \mathrm{~L}_{3} \quad(28.25 \%)$ and highest mortality percentage $(39.44 \%)$ was observed by interaction of $\mathrm{T}_{2} \mathrm{~L}_{1}$ i.e. (IBA $4000 \mathrm{ppm}$ and 10 $\mathrm{cm}$ length). However, the interaction effect of IBA concentrations, length and diameter of cuttings $(T \times D \times L)$ was found to be non significant on mortality percentage of pomegranate cuttings.

This might be due to the fact that, large sized cuttings might have got sufficient food material and hormones leading to more survival.

Similarly, it also might be due to better development of root system with good quality root and shoot parameters enabling the rooted cuttings to make better growth and there by accounted the lowest mortality at 90 DAP. Similar result was also reported by Sharma $e t$ al., (2009).

In conclusion, the pomegranate cuttings of 1.5 $\mathrm{cm}$ diameter and $20 \mathrm{~cm}$ length when treated with IBA $3000 \mathrm{ppm}$ concentration solution for 15- 20 second gives significantly highest success percentage of pomegranate cuttings $(70.12 \%)$ was observed in treatment $\mathrm{T}_{1}$ i.e. (IBA $3000 \mathrm{ppm}$ ), highest survival percentage $(68.59 \%)$ was recorded in treatment $\mathrm{T}_{1}$ i.e. (IBA $3000 \mathrm{ppm}$ ) and lowest mortality percentage $(31.40 \%)$ of pomegranate cuttings was observed in treatment $\mathrm{T}_{1}$ i.e. (IBA 3000 ppm). 


\section{References}

Abdelrahman S. and A. Al- Wasel, (1999). In vitro clonal propagation of Al- Belehi pomegranate (Punica granatum L.). Journal of King Saud University. Agricultural Science, 11: 3-14.

Hartman, Kester and Davies (1990). Plant propagation; principles and practices $5^{\text {th }}$ edition Prince-Hall, London, 727.

Hartman, Kester, Davies and Geneva (2002). Plant propagation; principles and practices $6^{\text {th }}$ edition Prince-Hall International editions, Englewood Cliffs, New Jersey, USA.

Kaur and Kaur., (2018). Determination of suitable cutting size for rooting of Pear cuttings $c v$. Patharnakh. International Journal of Agriculture Science, Vol. 10 (12), 6445-6448.

Leaky, R.R.B., Mohammed, H.R.S., (1985) The effect of stem length on root initiation in sequential single node cuttings of Triplochitonsleroxylon K. chum. J. Horticultu. Sci., 60(5), 431437
Levin, G.M. 2006. Pomegranate roads:a Soviet botanist's exile from Eden. pp. 15-183. B.L.Baer (ed.), Floreat Press, Forestville, CA.

Mar. M., 2000, Pomegranate plants materials. Genetics resources and breeding, a review options Mediterranean's series A. Seminnaires Mediterranean's, 42: 55-62.

Rana R.S. and Sood K.K. (2012). Effect of cutting diameter and hormonal application on the propagation of Ficus roxburghii Wall. through branch cuttings. Ann. For. Res. 55(1) 69-84. 2012

Sharma N., Anand R. and Kumar D. (2009). Standardization of pomegranate (Punica granatum L.) propagation through cuttings. Biological forum- An International Journal, 1(1): 75-80.

Sheikh, M. K and Rao, M, M. (2002). Effect of pruning and fruit load on yield and quality in pomegranate (Punica granatum L.) var Ganesh. Karanataka J. Agric. Sci.,15:3,549-555.

\section{How to cite this article:}

Basir Ahmad Ahmadi, G. M. Waghmare and Bhosale, A. M. 2021. Effect of IBA (Indole-3butyric acid) concentration on Success, Survival and Mortality of Pomegranate (Punica garanatum L.) Cutting. Int.J.Curr.Microbiol.App.Sci. 10(01): 965-974.

doi: https://doi.org/10.20546/ijcmas.2021.1001.117 